Memories II International

Congress Forests and

Agroforestry for the 21st

Century

Corresponding Author:

J. Freire Cruz

Published: 21 January 2021

Production and Hosting by

Knowledge E

(c) J. Freire Cruz et al. This article is distributed under the terms of the Creative Commons Attribution License, which permits unrestricted use and redistribution provided that the original author and source are credited.

\section{Mechanical Properties of Three-layer Boards of Pine (Pinus radiata) and Pigue (Piptocoma discolor) Owned by the PISMADE S.A. Com- pany}

\section{Propiedades Mecánicas de Tableros Alistona- dos Tricapa de Pino (Pinus radiata) y Pigue (Piptocoma discolor) Propiedad de la Empresa PISMADE S.A.}

\author{
J. Freire Cruz ${ }^{1}$, E. Salazar Castañeda ${ }^{2}$, V. Noboa ${ }^{2}$, and E. Cabezas ${ }^{1}$ \\ ${ }^{1}$ GIFOR, Grupo de Ingeniería Forestal, Chimborazo, Ecuador \\ ${ }^{2}$ Carrera de Ingeniería Forestal, Facultad de Recursos Naturales, Escuela Superior Politécnica \\ de Chimborazo, Chimborazo, Ecuador
}

\section{Abstract}

The present study proposes to determine the mechanical properties of three-layer boards of pine (Pinus radiata) and pigue (Piptocoma discolor) owned by the PISMADE S.A. Company. The tests were carried out under established technical procedures, with the use of international standards, at the Materials Resistance laboratory of the Faculty of Mechanics located in the Higher Polytechnic School of Chimborazo. 24 test pieces belonging to three-layer boards provided by the company were evaluated, each of them of different dimensions for bending and compression tests. It was possible to verify the different resistances that the boards of these species support; bending and compression values for both pine and pigue were obtained, appropriate for their use in the industry. The information generated deems this material rigid and appropriate for its efficient and durable use.

Keywords: compression, flexion, mechanical properties, wood boards.

\section{Resumen}

El presente estudio propone determinar las propiedades mecánicas en tableros tricapa de pino (Pinus radiata) y pigue (Piptocoma discolor) propiedad de la empresa PISMADE S.A. Los ensayos se los realizó bajo procedimientos técnicos, con la utilización de normas internacionales, dentro del laboratorio de Resistencia de Materiales de la facultad de Mecánica ubicado en la Escuela Superior Politécnica de Chimborazo. Evaluándose 24 probetas pertenecientes a tableros alistonados tricapa, cada una de ellas de diferentes dimensiones para ensayos de flexión y compresión, proporcionados por la empresa. Se pudo comprobar las diferentes resistencias que soportan los tableros de estas especies, obteniendo valores de flexión y compresión tanto para pino y pigue apropiados para su utilización en la industria. La información generada considera a este material rígido y apropiado para su uso eficiente y duradero.

Palabras Clave: compresión, flexión, propiedades mecánicas, tableros de madera. 


\section{Introducción}

En la actualidad en nuestro país, el uso de la madera como elemento estructural se ha visto con desconfianza e incertidumbre debido a la falta de normativas y conocimiento, lo que hace que se aprovechen pocas especies forestales. Al no conocer cuáles son las características de cada madera resulta imposible destinarlas a un uso apropiado y darles una aplicación industrial inmediata lo que permitiría ahorrar tiempo y esfuerzo en su transformación y utilización desde el punto de vista estructural para su posterior producción y comercialización [1]. Las investigaciones realizadas están dirigidas a efectuar el estudio de las propiedades físicas y mecánicas de especies forestales que pueden brindarnos mayores bondades el desarrollo industrial que se encuentra en pleno auge. Varias empresas están implementando nuevas líneas de producción, incrementando el campo de utilización de la madera especialmente como material de la construcción. La principal característica es su diferenciación individual, que hace que cada especie tenga un comportamiento distinto, en función de la cual tendrá diferentes usos. Es muy importante conocer estas características para

poder asegurar la resistencia que cada especie tendrá a las solicitaciones a las que será sometida [2]. La empresa PISMADE S.A. ha implementado la creación de nuevos tableros para la construcción de varias capas diseñados para el revestimiento de superficies exteriores e interiores, destacando a los tableros alistonados tricapa, formados por tres capas de madera, dos de ellas dispuestas en sentido longitudinal y la capa central en sentido transversal de tal manera que no pierda sus propiedades y su resistencia mecánica [3].

\section{Materiales y Métodos}

\subsection{Elaboración de las probetas para ensayos mecánicos}

De los tableros alistonados tricapa de pino (Pinus radiata) y pigue (Piptocoma discolor) de medidas $244 \times 122 \times 3,8 \mathrm{~cm}$ fabricados en la empresa PISMADE S.A. se realizaron 24 probetas de diferentes dimensiones para los ensayos de las propiedades mecánicas según especificaciones de la norma ASTM D143 [4], como es la determinación de la resistencia a la flexión longitudinal, resistencia a la flexión transversal, resistencia a la compresión longitudinal y resistencia a la compresión transversal.

\subsection{Ensayos mecánicos de Flexión}

La flexibilidad es la propiedad que tienen algunas maderas de poder ser dobladas o ser curvadas en su sentido longitudinal y transversal. Si son elásticas recuperan su forma primitiva cuando cesa la fuerza que las ha deformado. La madera presenta especial aptitud para sobrepasar su límite de elasticidad por flexión sin que se produzca rotura inmediata [5]. Las influencia directa que afecta a la resistencia a la flexión es la inclinación de la fibra [6]. 


\section{Table 1}

Características de la probetas de Pinus radiata y Piptocoma discolor para el estudio.

\begin{tabular}{|c|c|c|c|c|c|}
\hline Probeta & No. probetas & No. capas & Especie & Norma & Dimensiones \\
\hline \multirow[t]{2}{*}{$\begin{array}{l}\text { Flexión } \\
\text { longitudinal }\end{array}$} & 3 & 3 & $\begin{array}{l}\text { PIGUE } \\
\text { (Piptocoma } \\
\text { discolor) }\end{array}$ & ASTM D143 & $2,5 \times 2,5 \times 41 \mathrm{~cm}$ \\
\hline & 3 & 3 & $\begin{array}{l}\text { PINO (Pinus } \\
\text { radiata) }\end{array}$ & ASTM D143 & \\
\hline \multirow[t]{2}{*}{$\begin{array}{l}\text { Flexión } \\
\text { transversal }\end{array}$} & 3 & 3 & $\begin{array}{l}\text { PIGUE } \\
\text { (Piptocoma } \\
\text { discolor) }\end{array}$ & ASTM D143 & $2,5 \times 2,5 \times 41 \mathrm{~cm}$ \\
\hline & 3 & 3 & $\begin{array}{l}\text { PINO (Pinus } \\
\text { radiata) }\end{array}$ & ASTM D143 & \\
\hline \multirow[t]{2}{*}{$\begin{array}{l}\text { Compresión } \\
\text { longitudinal }\end{array}$} & 3 & 3 & $\begin{array}{l}\text { PIGUE } \\
\text { (Piptocoma } \\
\text { discolor) }\end{array}$ & ASTM D143 & $5 \times 5 \times 20 \mathrm{~cm}$ \\
\hline & 3 & 3 & $\begin{array}{l}\text { PINO (Pinus } \\
\text { radiata) }\end{array}$ & ASTM D143 & \\
\hline \multirow[t]{2}{*}{$\begin{array}{l}\text { Compresión } \\
\text { transversal }\end{array}$} & 3 & 3 & $\begin{array}{l}\text { PIGUE } \\
\text { (Piptocoma } \\
\text { discolor) }\end{array}$ & ASTM D143 & $5 \times 5 \times 15 \mathrm{~cm}$ \\
\hline & 3 & 3 & $\begin{array}{l}\text { PINO (Pinus } \\
\text { radiata) }\end{array}$ & ASTM D143 & \\
\hline
\end{tabular}

Para el procedimiento se colocaron las probetas en la máquina de ensayos universales tanto de manera longitudinal como de manera transversal de cada especie a estudiar (pino y pigue), la carga es aplicada al centro de manera gradual y continua, la longitud libre entre soportes debe ser igual a la mencionada en la norma utilizada ASTM D143 [4], las bases de cada uno de ellos deben lograr una posición horizontal exacta de la probeta a ensayar.

La carga se aplica continuamente a la probeta con una velocidad constante de la cabeza móvil de la prensa hidráulica más o menos, $1,5 \mathrm{~kg} / \mathrm{segundo}$. Se realiza por cada prueba una gráfica de carga contra deformación y se anotan todos los detalles que se consideren son importantes.

Es importante también la determinación del módulo de elasticidad a flexión.

\subsection{Ensayos mecánicos de Compresión}

Las probetas tanto de manera longitudinal como de manera transversal de cada especie estudiada (pino y pigue) se colocaron centradas en las quijadas: Mordaza o base de la máquina de ensayos universales y totalmente horizontales a las caras para evitar cualquier desviación o descentralización de la carga en cada una de las probetas. La carga es aplicada de forma continua a una velocidad constante, se toman las deformaciones por medio del compresómetro. 
Se determinó la carga y la deflexión para la primera falla, la carga máxima y puntos de cambio repentino. Se realizó la gráfica de carga contra deformación y se determinó el punto al límite elástico.

Se calculó el módulo de elasticidad a compresión y se obtuvo gráficamente considerando que es la pendiente de la porción recta de la curva de esfuerzo contra deformación unitaria.

\section{Resultados y Discusión}

\subsection{Flexión longitudinal}

En cuanto a la resistencia a la flexión longitudinal, la probeta que más fuerza soporto es la que corresponde al tablero alistonado tricapa de pino (P. radiata), con $340,79 \mathrm{~kg} / \mathrm{cm}^{2}$. De igual manera se obtuvo un valor mínimo de resistencia a la flexión longitudinal de $152,98 \mathrm{~kg} / \mathrm{cm}^{2}$ perteneciente al tablero de pigue ( $P$. discolor).

\section{Table 2}

Valores de flexión longitudinal de tableros alistonados tricapa para Pinus radiata y Piptocoma discolo.

Probeta no.
1
2
3
4
5
6

Especie
PIGUE (Piptocoma discolor)
PIGUE (Piptocoma discolor)
PIGUE (Piptocoma discolor)
PINO (Pinus radiata)
PINO (Pinus radiata)
PINO (Pinus radiata)

Resistencia a la flexión
longitudinal $\left(\mathbf{k g} / \mathbf{c m}^{2}\right)$
158,37
152,98
333,33
236,24
340,79
241,01

MOE longitudinal E
$\left[\mathbf{k g} / \mathbf{c m}^{2}\right]$
75333,05
71142,59
117715,38
36576,58
60533,79
29169,77

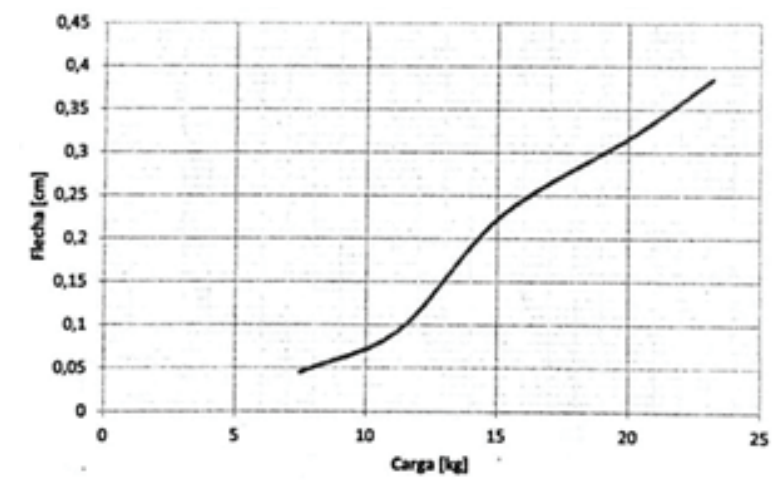

Figure 1

Carga deformación probeta 2. 


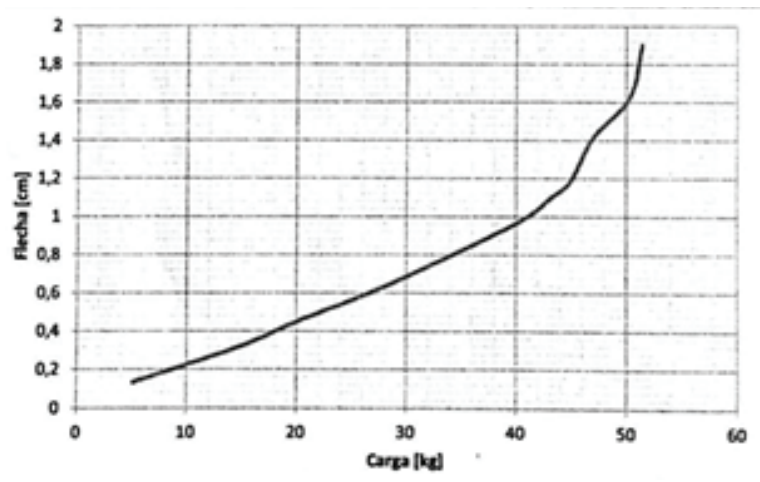

Figure 2

Gráfico carga deformación probeta 5.

\subsection{Flexión transversal}

En resistencia a la flexión transversal la probeta que más fuerza soporto es la que corresponde al tablero alistonado tricapa de pigue (P. discolor) con 505,10 kg/cm y como valor menor de $316,27 \mathrm{~kg} / \mathrm{cm}^{2}$ perteneciente al tablero de pino ( $P$. radiata).

Table 3

Valores de flexión transversal de tableros alistonados tricapa.

\begin{tabular}{|c|c|c|c|}
\hline PROBETA no. & Especie & $\begin{array}{l}\text { Resistencia a la flexión } \\
\text { transversal }\left(\mathrm{kg} / \mathrm{cm}^{2}\right)\end{array}$ & $\begin{array}{l}\text { MOE transversal E } \\
{\left[\mathrm{kg} / \mathrm{cm}^{2}\right]}\end{array}$ \\
\hline 1 & PIGUE (Piptocoma discolor) & 505,10 & 109240,91 \\
\hline 2 & PIGUE (Piptocoma discolor) & 410,49 & 69405,71 \\
\hline 3 & PIGUE (Piptocoma discolor) & 447,51 & 68744,05 \\
\hline 4 & PINO (Pinus radiata) & 386,03 & 55340,10 \\
\hline 5 & PINO (Pinus radiata) & 316,27 & 48462,16 \\
\hline 6 & PINO (Pinus radiata) & 366,83 & 65922,10 \\
\hline
\end{tabular}

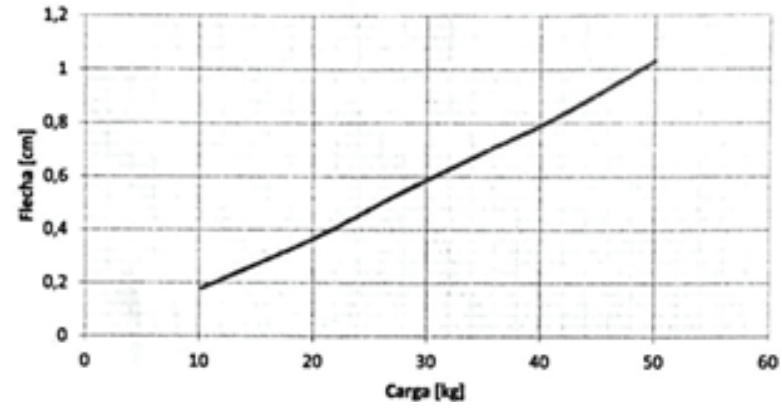

Figure 3

Gráfico carga deformación probeta 1. 


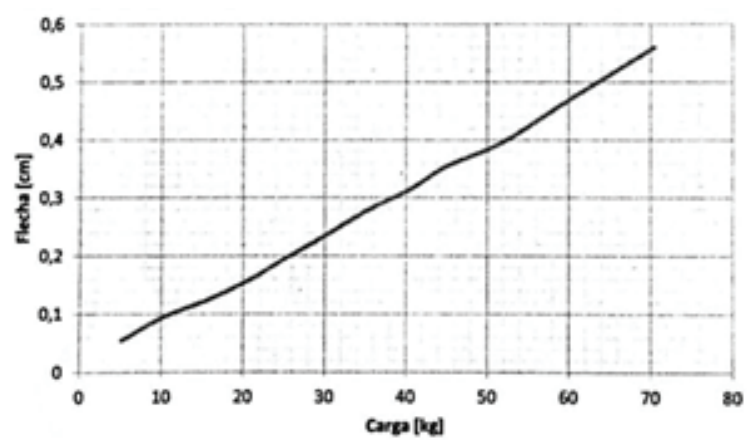

Figure 4

Gráfico carga deformación probeta 5.

\subsection{Compresión longitudinal}

Como resultado de la resistencia a la compresión longitudinal tenemos que la probeta que corresponde al tablero alistonado tricapa de pino ( $P$. radiata) soporta mayor fuerza que el resto con un valor de $61,52 \mathrm{~kg} / \mathrm{cm}^{2}$, como dato menor tenemos a $27,20 \mathrm{~kg} / \mathrm{cm}^{2}$ perteneciente al tablero de pigue ( $P$. discolor).

\section{Table 4}

Valores de compresión longitudinal de tableros alistonados tricapa.

Probeta no.
1
2
3
4
5
6

Especie
PIGUE (Piptocoma discolor)
PIGUE (Piptocoma discolor)
PIGUE (Piptocoma discolor)
PINO (Pinus radiata)
PINO (Pinus radiata)
PINO (Pinus radiata)

Resistencia a la compresión longitudinal $\left(\mathrm{kg} / \mathrm{cm}^{2}\right)$

27,79

30,76

27,20

58,44

61,52

61,32

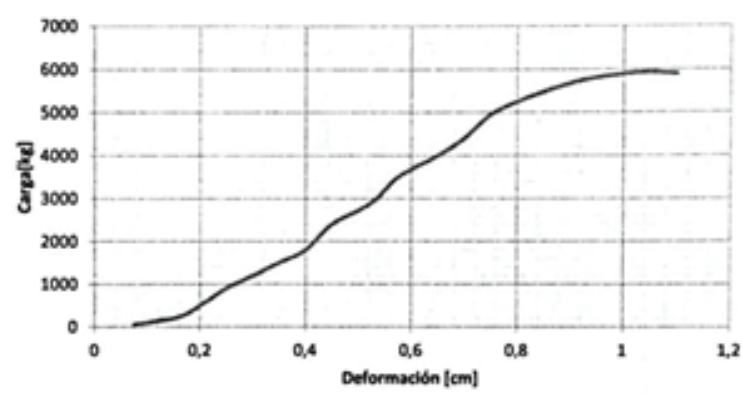

Figure 5

Gráfico carga deformación probeta 3. 


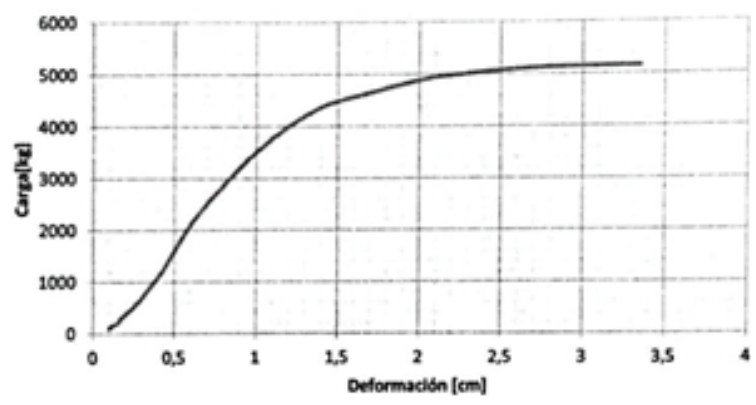

\section{Figure 6}

Gráfico carga deformación probeta 5.

\subsection{Compresión transversal}

La mayor resistencia a la compresión transversal lo presenta la probeta perteneciente al tablero alistonado tricapa de pino ( $P$. radiata) con un valor d $64,60 \mathrm{~kg} / \mathrm{cm}^{2}$ y la de menor valor de resistencia con un dato de $56,57 \mathrm{~kg} / \mathrm{cm}^{2}$ a la perteneciente al tablero de pigue ( $P$. discolor).

\section{Table 5}

Valores de compresión transversal de tableros alistonados tricapa.

Probeta no.
1
2
3
4
5
6

Especie
PIGUE (Piptocoma discolor)
PIGUE (Piptocoma discolor)
PIGUE (Piptocoma discolor)
PINO (Pinus radiata)
PINO (Pinus radiata)
PINO (Pinus radiata)

Resistencia a la compresión Transversal $\left(\mathbf{k g} / \mathrm{cm}^{2}\right)$

56,57

60,34

60,11

64,60

60,80

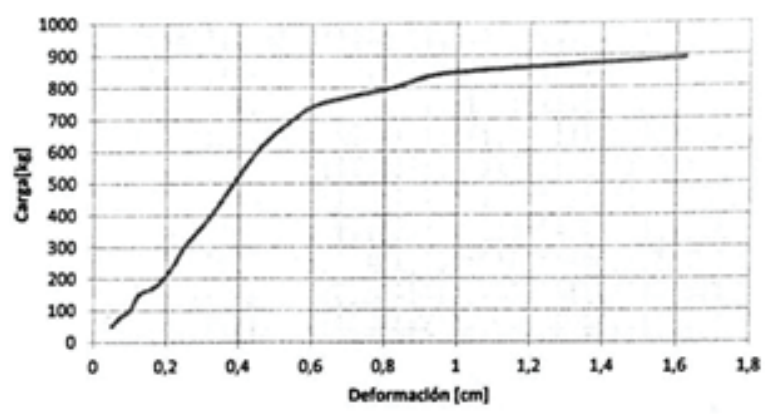

Figure 7

Carga deformación probeta 1. 


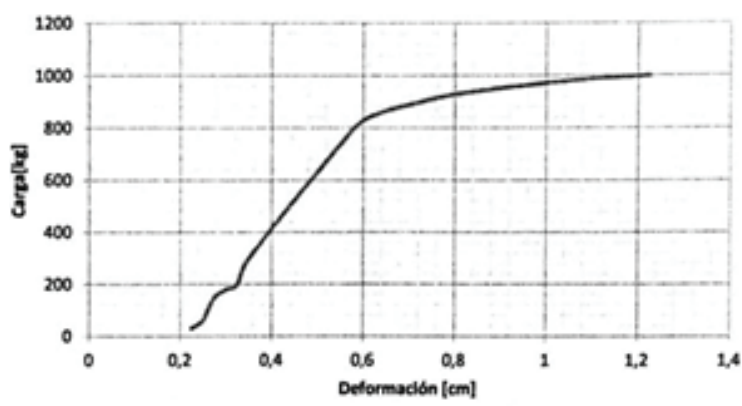

Figure 8

Gráfico carga deformación probeta 5.

\subsection{Valores de resistencia y rigidez característicos de tableros alis- tonados}

De acuerdo a los resultados (Tabla 6) de las características obtenidas en estudios de modelos de plancha en tableros multicapa de 3 y 5 capas de diferentes especies realizados por la empresa Binderholz $\mathrm{GmbH}$ [7] muestran valores similares a los obtenidos en los tableros tricapa de diferente grosor nominal elaborados en la empresa PISMADE S.A. Los ensayos mecánicos indican la resistencia arrojando los valores mostrados en la Tabla 7.

\section{Table 6}

Valores de resistencia de tableros alistonados empresa Binderholz $\mathrm{GmbH}$.

\begin{tabular}{|c|c|c|c|c|c|c|c|c|c|c|c|c|}
\hline \multicolumn{13}{|c|}{ Tablero de 3 y 5 capas empresa Binderholz GmbH } \\
\hline \multicolumn{13}{|c|}{ Carga sobre la plancha } \\
\hline $\mathrm{f} \mathrm{m}, \mathrm{O}$ & 37,0 & 34,9 & 31,6 & 30,3 & 28,7 & 24,4 & 23,8 & 26,3 & 24,2 & 21,9 & 23,1 & 20,5 \\
\hline $\mathrm{f} \mathrm{m}, 90$ & 6,7 & 6,5 & 8,1 & 7,3 & 6,4 & 11,4 & 11,9 & 9,2 & 11,5 & 13,8 & 12,5 & 15,1 \\
\hline $\mathrm{E} \mathrm{m}, 0$ & 11300 & 11400 & 10900 & 11000 & 11400 & 9700 & 9500 & 10500 & 9600 & 8700 & 9200 & 8200 \\
\hline $\mathrm{E} \mathrm{m}, 90$ & 1000 & 900 & 1450 & 1350 & 900 & 2600 & 2800 & 1800 & 2700 & 3600 & 3150 & 4150 \\
\hline
\end{tabular}

\section{Table 7}

Valores de resistencia de tableros alistonados empresa PISMADE S.A.

\begin{tabular}{|c|c|c|c|c|}
\hline \multicolumn{5}{|c|}{ Tableros tricapa empresa PISMADE S.A. } \\
\hline Resistencia $\mathbf{k g} / \mathrm{cm}^{2}$ & 240,79 & 505,10 & 61,52 & 64,60 \\
\hline Resistencia N/mm ${ }^{2}$ & 33,42 & 49,53 & 6,03 & 6,33 \\
\hline
\end{tabular}

\section{Conclusiones}

Los resultados de los ensayos muestran que las probetas de tableros tricapa de $P$. radiata en su mayoría son las que presentan mayor resistencia en comparación con los de la especie $P$. discolor, pero ambas aptas para su utilización industrial. 
Las probetas de tableros tricapa de $P$. discolor muestran algunas irregularidades debido a problemas presentados con los materiales, en este caso el pegamento entre cada una de las capas, lo cual impide obtener un valor más alto antes de la ruptura en sus fibras.

El comportamiento mecánico de los tableros tricapa de estas dos especies en estudio es congruente con el esperado y se considera adecuado para su uso en la industria. Los datos sugieren que se trata de un material rígido, apto para soportar esfuerzos cuando está sometido a flexión y compresión.

Se destaca el alto rendimiento de la madera de $P$. discolor en la fabricación de tableros tricapa, existe la posibilidad de la utilización de esta especie nativa no convencional por su rápido crecimiento y desarrollo en comparación con $P$. radiata, teniendo valores mecánicos de resistencia y rigidez apropiados para su utilización en la industria.

Los valores aseguran a los tableros tricapa para la fabricación y construcción en madera. Este material cumple condiciones técnicas que lo hacen estable y duradero. La técnica y el procedimiento de encolado proporcionan excelentes propiedades en relación a su resistencia.

Los resultados obtenidos nos dan una referencia muy importante para su utilización industrial y su comercialización, considerando a este material con mucha potencialidad en aplicaciones de alto valor agregado.

\section{References}

[1] Paguay I. Determinación de las propiedades físicas y mecánicas de tres especies forestales andinas: platuquero (Styloceras sp), yagual (Polylepis racemosa), nogal (Juglans neotropica) [Determination of the physical and mechanical properties of three Andean forest species: platuquero (Styloceras sp), yagual (Polylepis racemosa), walnut ( Juglans neotropica),Tesis de Grado. Ingeniera Forestal]. Riobamba - Ecuador: Escuela Superior Politécnica de Chimborazo; 2013. Spanish

[2] Umerez $\mathrm{H}$. Análisis de propiedades físicas de la madera de Populus $x$ euramericana $Y$ Pinus pinaster tras tratamientos de oleotermia [Analysis of physical properties of the wood of Populus $x$ euramericana and Pinus pinaster after oleothermic treatments,Tesis de Maestría. Ingeniero en Montes]. PalenciaEspaña: Universidad de Valladolid; 2015. Spanish

[3] Ecuador Forestal. Sector forestal productivo formal [Formal productive forestry sector]. 2019 [Citado el 10 Mar 2019]. Recuperado de: https://ecuadorforestal.org/informacion-s-f-e/ sector-forestal-productivo-formal/. Spanish

[4] American Society of Testing Materials. Standard Test Methods for Small Clear Specimens of Timber 1 ; 2014 [Citado el 20 Mar 2019]. Recuperado de: https://doi.org/10.1520/D0143-14.2. Spanish

[5] Pérez E. Análisis de las propiedades físico mecánicas para un sustituto de madera natural elaborado a base de plásticos reciclados [Analysis of the physical mechanical properties for a natural wood substitute made from recycled plastics,Tesis de Grado. Ingeniero Civil]. Guatemala: Universidad de San Carlos de Guatemala; 2010. Spanish

[6] Almeida J. (2015). Ensayo de Materiales [Material Testing,Internet]. Flexión; 2015 [Citado el 29 Oct 2019]. Recuperado de: https://prezi.com/aa7gzsqna99k/ensayo-de-materiales-flexion/. Spanish

[7] Binderholz GmbH. Tableros para la construcción [Boards for construction,Internet]. Tirol - Austria; 2018 [Citado 1 Nov 2019]. Recuperado de: https://www.binderholz.com/es/. Spanish 